\title{
Relationship of Trimester-Specific Maternal Thyroid Function With Gestational Weight Gain And Birth Weight In Pregnant Women With Normal Thyroid Autoimmunity
}

Xin He

Shanghai Municipal Center for Disease Control and Prevention

Zhengyuan Wang

Shanghai Municipal Center for Disease Control and Prevention

Zehuan Shi

Shanghai Municipal Center for Disease Control and Prevention

Ping Liao

Shanghai Municipal Center for Disease Control and Prevention

Qin Yan

Shanghai Municipal Center for Disease Control and Prevention

Qi Song

Shanghai Municipal Center for Disease Control and Prevention Xueying Cui

Shanghai Municipal Center for Disease Control and Prevention Wenjing Wang

Shanghai Municipal Center for Disease Control and Prevention

Jiajie Zang ( $\nabla$ zangjiajie@scdc.sh.cn )

Shanghai Municipal Center for Disease Control and Prevention

\section{Research Article}

Keywords: thyroid hormone, thyroid antibody, gestational weight gain, birth weight, trimester

Posted Date: September 8th, 2021

DOI: https://doi.org/10.21203/rs.3.rs-778871/v1

License: (9) (7) This work is licensed under a Creative Commons Attribution 4.0 International License. Read Full License 


\section{Abstract}

Background: Gestational weight gain (GWG) and birth weight are important indicators of maternal and infant health outcomes. Thyroid function may affect body composition and fetal growth during pregnancy. So far, on the premise of excluding thyroid autoimmunity interference, the correlation of thyroid function in different pregnancy with neonatal birth weight and maternal weight gain is still controversial.

Methods: This study used data from a prospective birth cohort in Shanghai, China. Serum concentration of TSH, total thyroxine (TT4), free thyroxine (FT4), total triiodothyronine (TT3), free triiodothyronine (FT3), thyroglobulin antibody (TGAb), thyroid peroxidase antibody (TPOAb) were determined using chemiluminescence assay. Maternal GWG and neonatal sex-specific birth weight-for-gestational age Z-score was calculated.

Results: Subjects with a higher BMI before pregnancy turned to have a lower GWG and an infant with a higher birth weight. GWG was negatively associated with maternal FT4 at the first and third trimester $(p<0.05)$, and TT4 at the third trimester $(p<0.05)$. GWG was positively associated with maternal FT3 at the second trimester $(p<0.05)$. Lower birth weight $\mathrm{Z}$-score were associated with higher FT4 at the second and third trimester ( $<<0.05)$, and higher TT4 at the second trimester $(p<0.05)$.

Conclusions: In the general pregnant population with normal thyroid autoimmunity, higher maternal FT4 and TT4 and lower FT3 are associated with less GWG. Higher maternal FT4 and TT4 have an infant with lower birth weight.

\section{Background}

Gestational weight gain (GWG) is an important indicator of maternal and fetal health outcomes [1, 2]. Excessive or insufficient GWG have been associated with higher risk of various unfavorable maternal and infant outcomes, such as gestational diabetes mellitus, exacerbation of asthma, large for gestational age (LGA), small for gestational age (SGA) and preterm birth [2-4]. The institute of Medicine (IOM) recommends maintaining proper GWG during pregnancy, taking into account pre-pregnancy BMI, to reduce the risk of adverse birth outcomes [5]. Birth weight could comprehensively reflect fetal development, nutrition and adaptation to intrauterine environments. It is considered to be an important indicator of fetal and postnatal health growth and development, and some studies have even shown that it may be associated with risks of chronic non-communicable diseases, such as obesity, diabetes mellitus in adulthood [6-8].

Thyroid hormones regulate body weight, body temperature, total and rest energy expenditure related to basal metabolic rate and body composition [9]. It was found that TSH correlates positively with body mass index (BMI), whereas total thyroxine (TT4) and free thyroxine (FT4) are negatively correlated with BMI in adults $[10,11]$. During pregnancy, the thyroid gland increases in size by $10 \%-40 \%$ with T3 and T4 synthesis increasing by nearly $50 \%$ [12]. Maternal weight gain and fetal birth weight could also be affected by maternal thyroid hormone-related changes. Previous studies showed maternal FT4 level was related to pregnancy BMI and GWG [13]. A prospective study of 430 mother-infant pairs found that maternal and neonatal free triiodothyronine (FT3) were also significantly associated with infant birth weight [14]. In a recent large prospective study of Chinese population, Zhou observed that maternal TSH and FT4 concentration were negatively associated with birth weight, while GWG may play a synergistic role [15]. Thus far, there is still controversy about which trimester and to what extent thyroid hormone plays effect on GWG and birth weight. Meanwhile, these studies almost ignored thyroid antibodies, although the incidence of TPOAb-positive or TGAb-positive during pregnancy reached 2-17\% and increased the risk of adverse birth outcomes [12, 16].

Therefore, the objective of the present study was to investigate the association between trimester-specific maternal thyroid function with GWG and birth weight in pregnant women with normal thyroid autoimmunity.

\section{Methods}

\subsection{Study design}

The present data was from a prospective birth cohort focusing on iodine nutritional status of pregnant women, thyroid function and the growth and development of their offspring in Shanghai, China, from April to October, 2017. A detailed description of the cohort is given in a previous paper [17]. This study was approved by the Ethical Committee of the Shanghai Center for Disease Control and Prevention (No. 2017-13). All participants provided written informed consents. The study enrolled 5485 pregnant women living in 16 districts of Shanghai over 6 months. The number of pregnant women fairly equally recruited from three trimesters (0-12 weeks, first trimester; 13-27 weeks, second trimester; and 28-40 weeks, third trimester). At enrollment, demographic data were collected using a face-to-face questionnaire interview. We excluded pregnant women with a prior history of diabetes, thyroid medication usage, thyroid disease, those treated using assisted reproductive, and twin pregnancies ( $n=303$ ); we also excluded those women lacking thyroid function test parameters or anthropometry data $(n=502)$, or outside the extreme value of thyroid function parameters $(n=56)$, as well as those with TPO-Ab positive or TgAb positive $(n=579)$. The final analytic sample thus comprised 4045 women.

\subsection{Maternal anthropometrics and GWG and birth weight Z-score}

Maternal anthropometrics were assessed at the recruitment visit. Maternal weight $(\mathrm{kg})$ and height $(\mathrm{cm})$ were determined without heavy clothing and shoes. Pre-pregnancy body mass index $(\mathrm{BMI})$ was calculated by dividing the self-reported pre-pregnancy weight $(\mathrm{kg})$ by the square of height $(\mathrm{m})(\mathrm{BMI}=\mathrm{weight}$ $[\mathrm{kg}] /$ height squared $\left.\left[\mathrm{m}^{2}\right]\right)$.

Subjects were categorized as underweight $\left(\mathrm{BMl}<18.5 \mathrm{~kg} / \mathrm{m}^{2}\right)$, normal weight $\left(\mathrm{BMl}=18.5-24.9 \mathrm{~kg} / \mathrm{m}^{2}\right)$, and overweight $/$ obese $\left(\mathrm{BMl}{ }^{3} 25.0 \mathrm{~kg} / \mathrm{m}^{2}\right) \mathrm{based}$ on their pre-pregnancy BMI (weight $(\mathrm{kg}) /$ height squared $\left(\mathrm{m}^{2}\right)$ ). Maternal postpartum weight, infant birth weight and gestational age at birth were obtained from medical records. Postpartum weight was measured just after delivery, and GWG=postpartum weight - pre-pregnancy weight [18]. Sex-specific birth weight-for- 
gestational age Z-score were established using the GAMLSS package in R [19]. Covariates information about maternal age, pre-pregnancy BMI, parity, maternal educational level and household income was assessed by questionnaires.

\subsection{Assessment of matemal thyroid function and urinary iodine}

Venous blood was acquired from consenting participants at enrollment. Coagulant vacuum tubes were centrifuged $10 \mathrm{~min}$ at $1800 \times \mathrm{g}$ and serum was separated and stored at $-80^{\circ} \mathrm{C}$ until testing. Serum levels of thyroid function parameters (TSH, FT3, FT4, TT4, TT3, TGAb, TPOAb) were detected by automatic luminescent immune analyzer (Cobas e 602; Roche., Switzerland or ADVIA Centaur XP; Siemens., German or DSI 800; Beckmen., the USA) at community medical service centers. The intra- and inter-assay coefficients of variation in all detection systems accorded to the clinical requirement. TPOAb positivity and TGAb positivity was defined as TPOAb $\geq 34 \mathrm{IU} / \mathrm{mL}$ or TgAb $\geq 115 \mathrm{IU} / \mathrm{mL}$ for Cobas e 602 , TPOAb $\geq 9 \mathrm{IU} / \mathrm{mL}$ or TgAb $\geq 4 \mathrm{IU} / \mathrm{mL}$ for DXI800, TPOAb $\geq 60 \mathrm{U} / \mathrm{mL}$ or $\mathrm{TgAb} \geq 60 \mathrm{U} / \mathrm{mL}$ for ADVIA Centaur XP. Spot urine sample were collected from pregnant women. The urinary iodine concentrations were determined using the acid digestion method ( $\mathrm{As}^{3+}-\mathrm{Ce}^{4+}$ catalytic spectrophotometry) in the Shanghai Municipal Center for Disease Prevention and Control.

\subsection{Statistical analyses}

Descriptive statistics were presented by pre-pregnancy BMI group. The demographic characteristics were presented as frequency [proportion (\%)]. GWG and birth weight were expressed as the mean [standard deviance (SD)], respectively.

Because of the deviation of reference ranges caused by the instruments, the data of the thyroid function in each research center cannot be analyzed directly. Therefore, the data of the thyroid function in each research center were divided into quartiles for subsequent statistical analysis and comparison. Multiple linear regression models were constructed using neonatal birth weight-Z score and GWG as dependent variables and single thyroid hormone quartiles as predictors. The lowest quartile of the thyroid hormone was used as the reference group. The initial crude regression models were subsequently adjusted for maternal age, gestational age at sampling, parity, maternal education, pre-pregnancy BMI, urinary iodine, and household income.

Data were analyzed using the R software (R Institute, Inc., Cary, NC, USA). A two-sided P value $£ 0.05$ was considered statistically significant.

\section{Results}

\subsection{Participants' characteristics}

Among the 4045 participants, 2845 pregnant women had a normal pre-pregnancy BMI (70.33\%). The study participants were primarily $26-30$ years old (43.85\%), pregnant with their firstborn (60.74\%). In addition, approximately half of the participants had a Bachelor's degree or higher (43.97\%), and many had a medium annual household income (59.26\%). Only three women reported cigarette smoking and about $1 \%$ of the women consumed alcohol during pregnancy. Subjects with a higher BMI before pregnancy turned to have a lower GWG and an infant with a higher birth weight. The GWG values were 8.36 \pm $5.80 \mathrm{~kg}, 7.06 \pm 1.96 \mathrm{~kg}$, and $4.22 \pm 3.74 \mathrm{~kg}$, and the neonatal birth weights were $3222.89 \pm 397.04 \mathrm{~g}, 3325.11 \pm 442.04 \mathrm{~g}$, and $3438.24 \pm 449.77 \mathrm{~g}$ for women categorized as pre-pregnancy underweight, normal weight, and overweight/obesity, respectively (Table 1). 
Table 1

Demographic, pregnancy and delivery characteristics of study participants, stratified by pre-pregnant body mass index (BMI)

\begin{tabular}{|c|c|c|c|c|c|c|c|c|c|}
\hline \multirow[t]{3}{*}{ Demorgraphic features } & \multicolumn{9}{|c|}{ Pre-pregnant BMI } \\
\hline & \multicolumn{2}{|l|}{ All } & \multicolumn{2}{|c|}{ Underweight } & \multicolumn{2}{|c|}{ Normal weight } & \multicolumn{2}{|c|}{ Overweight } & \multirow[t]{2}{*}{$P$} \\
\hline & $\mathrm{N}$ & $\% /$ Mean(sd) & $\mathrm{n}$ & $\% /$ Mean(sd) & $\mathrm{n}$ & $\% /$ Mean(sd) & $\mathrm{n}$ & $\% /$ Mean(sd) & \\
\hline Age & 4045 & & 523 & $28.25(4.13)$ & 2845 & 29.38(4.39) & 677 & $30.30(4.43)$ & \\
\hline $20 \sim 25$,yrs & 506 & $12.51 \%$ & 91 & $17.40 \%$ & 352 & $12.37 \%$ & 63 & $9.31 \%$ & \\
\hline $26 \sim 30$ & 1774 & $43.85 \%$ & 262 & $50.10 \%$ & 1256 & $44.15 \%$ & 256 & $37.81 \%$ & \\
\hline $31 \sim 35$ & 1225 & $30.28 \%$ & 127 & $24.28 \%$ & 850 & $29.88 \%$ & 248 & $36.63 \%$ & \\
\hline $36 \sim 40$ & 540 & $13.35 \%$ & 43 & $8.22 \%$ & 387 & $13.60 \%$ & 110 & $16.25 \%$ & \\
\hline Parity & & & 523 & & 2845 & & 677 & & \\
\hline firstborn & 2457 & $60.74 \%$ & 358 & $68.45 \%$ & 1727 & $60.70 \%$ & 372 & $54.95 \%$ & \\
\hline secondborn & 1588 & $39.26 \%$ & 165 & $31.55 \%$ & 1118 & $39.30 \%$ & 305 & $45.05 \%$ & \\
\hline Educational level & & & 521 & & 2841 & & 677 & & \\
\hline High school or less & 997 & $24.68 \%$ & 119 & $22.84 \%$ & 695 & $24.46 \%$ & 183 & $27.03 \%$ & \\
\hline College & 1266 & $31.34 \%$ & 150 & $28.79 \%$ & 875 & $30.80 \%$ & 241 & $35.60 \%$ & \\
\hline University or higher & 1776 & $43.97 \%$ & 252 & $48.37 \%$ & 1271 & $44.74 \%$ & 253 & $37.37 \%$ & \\
\hline Income & & & 520 & & 2837 & & 676 & & \\
\hline Low & 692 & $17.15 \%$ & 78 & $15 \%$ & 482 & $16.99 \%$ & 132 & $19.53 \%$ & \\
\hline Middle & 2391 & $59.26 \%$ & 303 & $58.27 \%$ & 1689 & $59.53 \%$ & 399 & $59.02 \%$ & \\
\hline High & 950 & $23.54 \%$ & 139 & $26.73 \%$ & 666 & $23.48 \%$ & 145 & $21.45 \%$ & \\
\hline Smoking habits & & & 521 & & 2838 & & 676 & & \\
\hline Never smoker & 3934 & $97.50 \%$ & 507 & $97.31 \%$ & 2779 & $97.92 \%$ & 648 & $95.86 \%$ & \\
\hline Stopped before pregnancy & 98 & $2.43 \%$ & 13 & $2.50 \%$ & 57 & $2.01 \%$ & 28 & $4.14 \%$ & \\
\hline Current smoker & 3 & $0.07 \%$ & 1 & $0.19 \%$ & 2 & $0.07 \%$ & 0 & $0 \%$ & \\
\hline Alcohol intake & & & 520 & & 2831 & & 675 & & \\
\hline Never drinker & 3611 & $89.70 \%$ & 473 & $90.96 \%$ & 2534 & $89.51 \%$ & 604 & $89.48 \%$ & \\
\hline Stopped before pregnancy & 373 & $9.26 \%$ & 37 & $7.12 \%$ & 270 & $9.54 \%$ & 66 & $9.78 \%$ & \\
\hline Current drinker & 42 & $1.04 \%$ & 10 & $1.92 \%$ & 27 & $0.95 \%$ & 5 & $0.74 \%$ & \\
\hline \multicolumn{10}{|l|}{ pregnant features } \\
\hline Gestational weight gain(kg) & 4041 & $6.75(4.86)$ & & $8.36(5.80)$ & & $7.06(4.96)$ & & $4.22(3.74)$ & \\
\hline Birth weight(g) & 4040 & $3328.04(463.07)$ & & 3222.89(397.04) & & $3325.11(442.04)$ & & $3438.24(449.77)$ & \\
\hline
\end{tabular}

\subsection{Association between trimester-specific quartiles of thyroid hormone and GWG}

Table 2 shows the association between trimester-specific quartiles of thyroid hormones and GWG. For the first and third trimester, there was a substantially inverse association between maternal FT4 and GWG. After adjustment for covariates, GWG was lower for participants with an FT4 level in the 4th quartile $(B=-1.45 \mathrm{~kg}, 95 \% \mathrm{Cl}:-2.48,-0.42])$ compared with that in the1st quartile at the first trimester $(p=0.01)$. At the third trimester, GWG was lower for participants with an FT4 level in the 2 nd quartile ( $B=-1.27 \mathrm{~kg}, 95 \% \mathrm{Cl}:-2.03,-0.51])$ and 3rd quartile $(B=-1.10 \mathrm{~kg}, 95 \% \mathrm{Cl}:-1.95,-0.24])$ compared with that in the1st quartile $(p<0.001, p=0.01)$. A significant inverse association for TT4 and GWG was observed at the third trimester $(Q 2 \mathrm{vs}$. Q1: $B=-0.92 \mathrm{~kg}, 95 \% \mathrm{Cl}$ : $-1.77,-0.07, p=0.03$; Q3 vs. Q1: $\beta=-1.18$ kg, 95\%Cl: $-2.06,-0.30, p=0.01 ; Q 4$ vs. Q1: $ß=-1.67 \mathrm{~kg}, 95 \% \mathrm{Cl}:-2.54,-0.81, \mathrm{p}<0.001)$. We detected a significant positive association for FT3 with GWG at the second trimester. Increased GWG was observed in the 2nd quartile ( $B=0.81 \mathrm{~kg}, 95 \% \mathrm{Cl}: 0.02,1.61, \mathrm{p}=0.04)$ and the 4th quartile ( $B=1.44 \mathrm{~kg}, 95 \% \mathrm{Cl}: 0.59,2.28, p<0.001)$ for FT3 compared with that in the 1 st quartile. No other thyroid function parameters were associated with GWG. 
Table 2

Multivariate associations between maternal thyroid hormone quartiles and gestational weight gain, stratified by trimesters

\begin{tabular}{|c|c|c|c|c|c|c|c|c|c|}
\hline & & \multicolumn{3}{|l|}{ First trimester } & \multicolumn{3}{|l|}{ Second trimester } & \multicolumn{2}{|l|}{ Third trimester } \\
\hline & & Crude & Adjusted & $\mathrm{p}$ & Crude & Adjusted & $\mathrm{p}$ & Crude & Adjusted \\
\hline & & $\beta(95 \% \mathrm{Cl})$ & $\beta(95 \% \mathrm{Cl})$ & & $\beta(95 \% \mathrm{Cl})$ & $\beta(95 \% \mathrm{Cl})$ & & $\beta(95 \% \mathrm{Cl})$ & $\beta(95 \% \mathrm{Cl})$ \\
\hline \multirow[t]{4}{*}{ TSH } & $\begin{array}{l}\text { Quartile } \\
1\end{array}$ & 0.00 & 0.00 & & 0.00 & 0.00 & & 0.00 & 0.00 \\
\hline & $\begin{array}{l}\text { Quartile } \\
2\end{array}$ & $0.48(-0.30,1.26)$ & $0.43(-0.35,1.21)$ & 0.28 & $0.30(-0.53,1.13)$ & $0.08(-0.73,0.90)$ & 0.84 & $-0.15(-1.10,0.80)$ & $-0.10(-1.05,0.85)$ \\
\hline & $\begin{array}{l}\text { Quartile } \\
3\end{array}$ & $0.54(-0.23,1.32)$ & $0.51(-0.28,1.30)$ & 0.21 & $-0.01(-0.84,0.82)$ & $-0.25(-1.07,0.58)$ & 0.55 & $2.44(-0.69,1.19)$ & $0.26(-0.68,1.20)$ \\
\hline & $\begin{array}{l}\text { Quartile } \\
4\end{array}$ & $0.44(-0.39,1.26)$ & $0.46(-0.36,1.29)$ & 0.27 & $0.06(-0.78,0.90)$ & $-0.09(-0.92,0.74)$ & 0.83 & $2.30(-0.61,1.20)$ & $0.18(-0.73,1.09)$ \\
\hline \multirow[t]{4}{*}{ FT3 } & $\begin{array}{l}\text { Quartile } \\
1\end{array}$ & 1.00 & 1.00 & & 1.00 & 1.00 & & 1.00 & 1.00 \\
\hline & $\begin{array}{l}\text { Quartile } \\
2\end{array}$ & $0.19(-0.89,1.27)$ & $0.35(-0.71,1.42)$ & 0.52 & $0.52(-0.28,1.33)$ & $0.81(0.02,1.61)$ & 0.04 & $0.13(-0.62,0.89)$ & $0.41(-0.35,1.16)$ \\
\hline & $\begin{array}{l}\text { Quartile } \\
3\end{array}$ & $0.38(-0.63,1.39)$ & $0.65(-0.36,1.66)$ & 0.21 & $0.17(-0.65,0.99)$ & $0.65(-0.16,1.47)$ & 0.12 & $0.66(-0.17,1.51)$ & $0.69(-0.15,1.54)$ \\
\hline & $\begin{array}{l}\text { Quartile } \\
4\end{array}$ & $-0.44(-1.41,0.51)$ & $-0.06(-1.04,0.91)$ & 0.90 & $0.53(-0.31,1.38)$ & $1.44(0.59,2.28)$ & $\begin{array}{l}<.001 \\
0.00\end{array}$ & $0.73(-0.33,1.79)$ & $0.92(-0.16,1.99)$ \\
\hline \multirow[t]{4}{*}{ FT4 } & $\begin{array}{l}\text { Quartile } \\
1\end{array}$ & 1.00 & 1.00 & & 1.00 & 1.00 & & 1.00 & 1.00 \\
\hline & $\begin{array}{l}\text { Quartile } \\
2\end{array}$ & $-0.18(-1.36,1.00)$ & $-0.18(-1.34,0.98)$ & 0.76 & $-0.02(-0.79,0.75)$ & $-0.34(-1.10,0.42)$ & 0.38 & $-1.14(-1.89,-0.38)$ & $-1.27(-2.03,-0.51)$ \\
\hline & $\begin{array}{l}\text { Quartile } \\
3\end{array}$ & $-0.91(-1.99,0.16)$ & $-0.93(-2.00,0.14)$ & 0.09 & $-0.20(-1.00,0.59)$ & $-0.55(-1.34, .0 .23)$ & 0.17 & $-1.04(-1.90,-0.19)$ & $-1.10(-1.95,-0.24)$ \\
\hline & $\begin{array}{l}\text { Quartile } \\
4\end{array}$ & $-1.00(-2.01,0.03)$ & $-1.45(-2.48,-0.42)$ & 0.01 & $0.28(-0.60,1.17)$ & $-0.14(-1.02,0.73)$ & 0.74 & $-0.44(-1.49,0.60)$ & $-0.58(-1.63,0.45)$ \\
\hline \multirow[t]{4}{*}{ TT3 } & $\begin{array}{l}\text { Quartile } \\
1\end{array}$ & 1.00 & 1.00 & & 1.00 & 1.00 & & 1.00 & 1.00 \\
\hline & $\begin{array}{l}\text { Quartile } \\
2\end{array}$ & $-0.30(-1.05,0.43)$ & $-0.10(-0.85,0.64)$ & 0.78 & $-0.45(-1.37,0.45)$ & $-0.16(-1.05,0.74)$ & 0.73 & $-0.47(-1.39,0.43)$ & $-0.46(-1.37,0.44)$ \\
\hline & $\begin{array}{l}\text { Quartile } \\
3\end{array}$ & $0.47(-0.30,1.26)$ & $0.71(-0.08,1.51)$ & 0.08 & $0.50(-0.37,1.37)$ & $0.81(-0.04,1.67)$ & 0.06 & $-0.71(-1.63,0.20)$ & $-0.64(-1.56,0.27)$ \\
\hline & $\begin{array}{l}\text { Quartile } \\
4\end{array}$ & $-0.39(-1.26,0.48)$ & $0.48(-0.40,1.37)$ & 0.29 & $-0.21(-1.05,0.64)$ & $0.82(-0.03,1.67)$ & 0.06 & $-0.35(-1.25,0.54)$ & $0.11(-0.80,1.02)$ \\
\hline \multirow[t]{4}{*}{ TT4 } & $\begin{array}{l}\text { Quartile } \\
1\end{array}$ & 1.00 & 1.00 & & 1.00 & 1.00 & & 1.00 & 1.00 \\
\hline & $\begin{array}{l}\text { Quartile } \\
\end{array}$ & $0.15(-0.67,0.96)$ & $0.07(-0.74,0.88)$ & 0.87 & $-0.30(-1.16,0.55)$ & $-0.64(-1.48,0.20)$ & 0.13 & $-0.64(-1.49,0.20)$ & $-0.92(-1.77,-0.07)$ \\
\hline & $\begin{array}{l}\text { Quartile } \\
3\end{array}$ & $\begin{array}{l}-0.44(-1.23 \\
0.36)\end{array}$ & $-0.48(-1.28,0.32)$ & 0.24 & $0.12(-0.72,0.96)$ & $-0.09(-0.92,0.74)$ & 0.83 & $-1.00(-1.87,-0.13)$ & $-1.18(-2.06,-0.30)$ \\
\hline & $\begin{array}{l}\text { Quartile } \\
4\end{array}$ & $-0.20(-1.02,0.61)$ & $-0.15(-0.97,0.66)$ & 0.71 & $-0.07(-0.91,0.76)$ & $-0.41(-1.24,0.41)$ & 0.32 & $-1.69(-2.55,-0.83)$ & $-1.67(-2.54,-0.81)$ \\
\hline
\end{tabular}

a Abbreviations: TSH, thyroid stimulating hormone; TT4, total thyroxine; FT4: free thyroxine; TT3, total triiodothyronine; FT3, free triiodothyronine; TRAb, thyrotrophin receptor antibody; TPOAb, thyroid peroxidase antibody; TGAb, anti-thyroglobulin.

b Model was adjusted for age, parity, maternal education, pre-pregnancy BMI, urinary iodine, household income and gestational age.

c Bold red text indicates $\mathrm{P}<0.05$.

\subsection{Association between birth weight Z-score and trimester-specific quartiles of thyroid hormones}

As shown in Table 3, multivariate analysis revealed a clear negative association between FT4 and birth weight Z-score in the second and third trimesters. At the second trimester, mothers with the $2 \mathrm{nd}$, 3rd, and 4th quartile of FT4 levels were associated with lower birth weight Z-score of -0.14 ( $95 \%$ Cl: $-0.01,-0.28, p=$ $0.04),-0.19(95 \% \mathrm{Cl}:-0.33,-0.05, \mathrm{p}=0.01)$, and $-0.19(95 \% \mathrm{Cl}:-0.35,-0.03, \mathrm{p}=0.05)$, respectively, compared with mothers with the 1 st quartile of FT4 levels.

Page 5/9 
The mothers with the 2 nd quartile of FT4 levels were associated with lower birth weight Z-score of $-0.12(95 \% \mathrm{Cl}:-0.25,-0.01, \mathrm{p}=0.05)$ at the third trimester. Additionally, the birth weight Z-score of the newborns of mothers with the 4th quartile of total T4 levels was associated with a decrease in birth weight Z-score of $-0.15(95 \% \mathrm{Cl}:-0.30,-0.01, \mathrm{p}=0.04)$ compared with that of the 1 st quartile of total T4 levels at the second trimester. Other maternal thyroid function parameters showed no statistically significant associations with neonatal birth weight.

Table 3

Multivariate associations between maternal thyroid hormone quartiles and birth weight, stratified by trimesters

\begin{tabular}{|c|c|c|c|c|c|c|c|c|c|c|}
\hline & & First trimester & & & Second trimester & & & Third trimester & & \\
\hline & & Crude & Adjusted & $\mathrm{p}$ & Crude & Adjusted & $\mathrm{p}$ & Crude & Adjusted & $\mathrm{F}$ \\
\hline \multirow[t]{4}{*}{ TSH } & $\begin{array}{l}\text { Quartile } \\
1\end{array}$ & 1.00 & 1.00 & & 1.00 & 1.00 & & 1.00 & 1.00 & \\
\hline & $\begin{array}{l}\text { Quartile } \\
2\end{array}$ & $-0.01(-0.15,0.14)$ & $0.03(-0.10,0.15)$ & 0.68 & $-0.05(-0.22,0.10)$ & $-0.08(-0.23,0.06)$ & 0.25 & $0.08(-0.08,0.25)$ & $0.07(-0.08,0.23)$ & C \\
\hline & $\begin{array}{l}\text { Quartile } \\
3\end{array}$ & $0.09(-0.05,0.24)$ & $0.07(-0.05,0.20)$ & 0.26 & $0.06(-0.09,0.22)$ & $-0.01(-0.15,0.14)$ & 0.99 & $0.02(-0.14,0.18)$ & $0.00(-0.15,0.15)$ & C \\
\hline & $\begin{array}{l}\text { Quartile } \\
4\end{array}$ & $0.06(-0.10,0.21)$ & $0.07(-0.06,0.20)$ & 0.32 & $0.01(-0.14,0.18)$ & $-0,04(-0.19,0.10)$ & 0.54 & $0.04(-0.11,0.20)$ & $0.03(-0.12,0.17)$ & $\mathrm{C}$ \\
\hline \multirow[t]{4}{*}{ FT3 } & $\begin{array}{l}\text { Quartile } \\
1\end{array}$ & 1.00 & 1.00 & & 1.00 & 1.00 & & 1.00 & 1.00 & \\
\hline & $\begin{array}{l}\text { Quartile } \\
2\end{array}$ & $-0.07(-0.28,0.13)$ & $-0.03(-0.21,0.14)$ & 0.67 & $0.01(-0.14,0.17)$ & $0.01(-0.13,0.16)$ & 0.87 & $0.09(-0.03,0.22)$ & $0.07(-0.04,0.19)$ & C \\
\hline & $\begin{array}{l}\text { Quartile } \\
3\end{array}$ & $0.01(-0.17,0.21)$ & $0.08(-0.08,0.24)$ & 0.35 & $0.11(-0.04,0.27)$ & $0.09(-0.05,0.24)$ & 0.22 & $0.09(-0.05,0.23)$ & $0.05(-0.08,0.19)$ & C \\
\hline & $\begin{array}{l}\text { Quartile } \\
4\end{array}$ & $0.12(-0.06,0.30)$ & $0.14(-0.02,0.30)$ & 0.09 & $0.12(-0.04,0.29)$ & $0.06(-0.09,0.21)$ & 0.45 & $0.14(-0.04,0.32)$ & $0.02(-0.15,0.19)$ & C \\
\hline \multirow[t]{4}{*}{ FT4 } & $\begin{array}{l}\text { Quartile } \\
1\end{array}$ & 1.00 & 1.00 & & 1.00 & 1.00 & & 1.00 & 1.00 & \\
\hline & $\begin{array}{l}\text { Quartile } \\
2\end{array}$ & $0.12(-0.10,0.34)$ & $0.17(-0.01,0.36)$ & 0.08 & $-0.16(-0.31,-0.01)$ & $-0.14(-0.28,-0.01)$ & 0.04 & $-0.12(-0.25,0.01)$ & $-0.12(-0.25,-0.01)$ & c \\
\hline & $\begin{array}{l}\text { Quartile } \\
3\end{array}$ & $0.03(-0.17,0.24)$ & $0.12(-0.05,0.29)$ & 0.19 & $-0.22(-0.37,-0.06)$ & $-0.19(-0.33,-0.05)$ & 0.01 & $-0.13(-0.27,0.02)$ & $-0.12(-0.26,0.01)$ & C \\
\hline & $\begin{array}{l}\text { Quartile } \\
4\end{array}$ & $0.07(-0.13,0.26)$ & $0.16(-0.01,0.33)$ & 0.06 & $-0.28(-0.45,-0.10)$ & $-0.19(-0.35,-0.03)$ & 0.02 & $-0.13(-0.31,0.04)$ & $-0.09(-0.26,0.07)$ & C \\
\hline \multirow[t]{4}{*}{ TT3 } & $\begin{array}{l}\text { Quartile } \\
1\end{array}$ & 1.00 & 1.00 & & 1.00 & 1.00 & & 1.00 & 1.00 & \\
\hline & $\begin{array}{l}\text { Quartile } \\
2\end{array}$ & $0.03(-0.11,0.17)$ & $0.06(-0.06,0.18)$ & 0.35 & $0.01(-0.17,0.18)$ & $-0.05(-0.21,0.11)$ & 0.51 & $-0.11(-0.26,0.04)$ & $-0.07(-0.21,0.08)$ & C \\
\hline & $\begin{array}{l}\text { Quartile } \\
3\end{array}$ & $-0.01(-0.16,013)$ & $-0.01(-0.13,0.12)$ & 0.94 & $0.07(-0.10,0.24)$ & $0.05(-0.10,0.21)$ & 0.52 & $0.09(-0.06,0.250$ & $0.07(-0.07,0.22)$ & C \\
\hline & $\begin{array}{l}\text { Quartile } \\
4\end{array}$ & $0.00(-0.16,0.16)$ & $0.05(-0.09,0.20)$ & 0.48 & $0.11(-0.05,0.28)$ & $0.04(-0.11,0.20)$ & 0.58 & $0.14(-0.01,0.30)$ & $0.05(-0.09,0.20)$ & C \\
\hline \multirow[t]{4}{*}{ TT4 } & $\begin{array}{l}\text { Quartile } \\
1\end{array}$ & 1.00 & 1.00 & & 1.00 & 1.00 & & 1.00 & 1.00 & \\
\hline & $\begin{array}{l}\text { Quartile } \\
2\end{array}$ & $-0.03(-0.18,0.12)$ & $0.05(-0.08,0.19)$ & 0.44 & $-0.08(-0.25,0.08)$ & $-0.04(-0.19,0.11)$ & 0.59 & $-0.14(-0.29,-0.01)$ & $-0.05(-0.19,0.09)$ & C \\
\hline & $\begin{array}{l}\text { Quartile } \\
3\end{array}$ & $-0.07(-0.22,0.08)$ & $-0.00(-0.13,0.13)$ & 0.10 & $-0.14(-0.31,0.02)$ & $-0.12(-0.27,0.02)$ & 0.10 & $-0.20(-0.35,-0.05)$ & $-0.12(-0.26,0.02)$ & C \\
\hline & $\begin{array}{l}\text { Quartile } \\
4\end{array}$ & $-0.00(-0.15,0.15)$ & $0.04(-0.09,0.17)$ & 0.57 & $-0.20(-0.36,-0.04)$ & $-0.15(-0.30,-0.01)$ & 0.04 & $-0.09(-0.24,0.05)$ & $-0.07(-0.21,0.07)$ & C \\
\hline
\end{tabular}

a Abbreviations: TSH, thyroid stimulating hormone; TT4, total thyroxine; FT4: free thyroxine; TT3, total triiodothyronine; FT3, free triiodothyronine; TRAb, thyrotrophin receptor antibody; TPOAb, thyroid peroxidase antibody; TGAb, anti-thyroglobulin.

b Model was adjusted for age, parity, maternal education, pre-pregnancy BMI, household income and gestational age.

c Bold red text indicates $\mathrm{P}<0.05$.

\section{Discussion}


In this large prospective study, on the premise of thyroid antibody-negative pregnant women, we found that maternal GWG was negatively correlated with FT4 level at the first and third trimester, TT4 level at the third trimester $(p<0.05)$, and positively correlated with FT3 level at the second trimester $(p<0.05)$. Pregnant women with higher FT4 at the second and third trimester and higher TT4 at the second trimester would have an infant with lower birth weight Z-score ( $p<$ $0.05)$.

Compared with several previous studies that showed FT4 in early pregnancy is inversely related to GWG, our study found that the third trimester is also the key period for FT4 and TT4 is to affect GWG $[13,20,21]$. FT3 in the second trimester of pregnancy was also positively correlated with GWG. We did not observe an association between TSH and maternal weight in all trimesters, which is in line with the results of the FaSTER Trial among 9209 euthyroid pregnant women [21]. However, a previous study involving 1035 Dutch Caucasian pregnant women and the Generation R study of 5206 mothers mainly Dutch origin showed that in early pregnancy, higher maternal TSH level was associated with an increased risk of excessive GWG [13, 20]. These inconsistencies may be caused by ethnic differences in the study population and TSH has a significant effect on gestational weight gain in the Dutch population cohort. Another possibility is that due to the stimulation of TSH receptor by hCG, the TSH level decreased significantly in early pregnancy, so the variations in gestational weeks at sampling time and detection methods can also cause differences in the analysis results.

Maternal subclinical hypothyroidism is associated with a higher risk of SGA and lower birth weight, whereas isolated hypothyroxinaemia is associated with lower risk of SGA and higher birth weight [22]. Previous studies also found an inverse correlation between TSH and birth weight in euthyroid pregnant women $[23,24]$. Significant inverse associations between birth weight and maternal FT4 in early pregnancy were reported [25]. The present study further showed that the second and third trimesters are the critical period during which maternal FT4 and TT4 affect birth weight. This is consistent with the rule that fetuses gain weight mainly after 24 weeks of gestation [26]. However, our study did not find the association between TSH and birth weight, which may be because TSH was detected by different instruments and was not analyzed as a continuous variable. Maternal FT4 and TT4's influence on intrauterine growth and development might act via maternal and/or placental mechanisms. The direct impact might be due shifts in the placental FT4 concentration playing a causal role in affecting the fetal bioavailability of growth factors (e.g., insulin like growth factors) and growth-related hormones (e.g., prostaglandins and growth hormones), and the stimulation of fetal metabolism (i.e., the consumption of glucose and oxygen), resulting in the induction of a direct catabolicstate in the fetus [27]. Alternatively, an indirect mechanism might be that higher maternal FT4 levels increase lipid and protein degradation, and enhance insulin sensitivity $[28,29]$, which results in a state of chronic caloric deficiency in the mother, producing a negative feedback on birth weight [30, 31].

Our study has several strengths, firstly, this was a large sample size of mother and baby pairs and participants were almost equally distributed from the first, second, and third trimesters of pregnancy. Secondly, our study comprehensively tested thyroid function and excluded interference of TPOAb-positive and TGAb-positive pregnant women. In women who were positive for TPOAb, TGAb, or both, TSH levels increased as gestation progressed, even hypothyroidism may occur because of the stress of pregnancy [32,33]. In 2017, new guidelines of American Thyroid Association (ATA) recommended that euthyroid pregnant women with positive TPOAb or TGAb should measure serum TSH level every 4 weeks from the confirmation of pregnancy to the mid-pregnancy [12]. Thirdly, our analysis carefully included several key confounding factors, such as pre-pregnancy BMI [34, 35], that have been largely ignored by previous studies. For example, we include urinary iodine level which may reflect iodine intake and iodine nutrition status. The present study had several limitations. First, thyroid function parameters were measured by different community medical centers and instruments, so we only modeled quartiles of thyroid parameters replacing direct measurement data for statistical analysis. In the future, we may analyze the data generated by the same instrument as continuous variables. Second, pre-pregnancy weight was self-reported thus might lead to underestimation and misclassification. Third, the crosssectional design of this study meant that we could not draw conclusions about causes and directions of the observed associations.

\section{Conclusion}

Higher maternal FT4 and TT4 levels are associated with less GWG lower birth weight when maternal thyroid parameters were modeled as quartiles.

\section{Abbreviations}

GWG gestational weight gain

LGA large for gestational age

SGA small for gestational age

TT4 total thyroxine

FT4 free thyroxine

TT3 total triiodothyronine

FT3 free triiodothyronine

TGAb hyroglobulin antibody

TPOAb thyroid peroxidase antibody

SD tandard deviance

BMI body mass index 


\section{Declarations}

Ethics approval and consent to participate: All methods were performed in accordance with the relevant guidelines and regulations (Declaration of Helsinki). This study was approved by the Ethical Committee of the Shanghai Center for Disease Control and Prevention (No. 2017-13). All participants provided written informed consents.

Consent for publication: The authors provide consents for publication.

Availability of data and materials: The datasets generated and/or analysed during the current study are not publicly available but are available from the corresponding author on reasonable request.

Competing interests: The authors declare that they have no competing interests

Funding: The current study was supported by the young fund of Shanghai municipal health commission (20194Y0443), food nutrition research and education fund of Danone nutrition center (DIC2019-03), excellent young talents of health system in Shanghai (No. 2017YQ043) and top young talents in Shanghai (No. 2020-8).

Authors' contributions : Xin He, article design and writing; Zhengyuan Wang, Zehuan Shi, Qi Song and Xueying Cui, cohort establishment and management; Ping Liao and Qin Yan, detection and quality control; Wenjing Wang and Jiajie Zang, article review.

Acknowledgments: We thank the study subjects and their families for their participation. The authors also thank the investigators from centers for disease control and prevention and community health service centers.

\section{References}

1. Caughey AB. Gestational Weight Gain and Outcomes for Mothers and Infants. JAMA. 2017, 317, 2175-2176.

2. Ali Z, Nilas L, Ulrik CS. Excessive gestational weight gain in first trimester is a risk factor for exacerbation of asthma during pregnancy: A prospective study of 1283 pregnancies. J. Allergy Clin. Immun. 2018, 141, 761-767.

3. Goldstein RF, Abell SK, Ranasinha S, Misso M, Boyle JA, Black MH, et al. Association of Gestational Weight Gain With Maternal and Infant Outcomes. JAMA. 2017, 317, 2207.

4. Voerman E, Santos S, Inskip H, Amiano P, Barros H, Charles M, et al. Association of Gestational Weight Gain With Adverse Maternal and Infant Outcomes. JAMA. 2019, 321, 1702.

5. 5.. Rasmussen K, Yaktine AL (editor). Weight gain during pregnancy: reexamining the guidelines. Danvers: Institute of Medicine (2009).

6. Tanya D, Mary K. Low birth weight: will new estimates accelerate progress? Lancet Glob Health, 2019, 77(7), e809-e810.

7. Stuart A, Amer-Wahlin I, Persson J, Kallen K. Long-term cardiovascular risk in relation to birth weight and exposure to maternal diabetes mellitus. Int $\mathrm{J}$ Cardiol 2013, 168, 2653-2657.

8. Khambalia AZ, Algert CS, Bowen JR, Collie RJ, Roberts CL. Long-term outcomes for large for gestational age infants born at term. J Paediatr Child Health 2017,53,876-881.

9. Reinehr, T. Obesity and thyroid function. Mol. Cell. Endocrinol. 2010, 316, 165-171.

10. Biondi, B. Thyroid and Obesity: An Intriguing Relationship. The Journal of Clinical Endocrinology \& Metabolism. 2010, 95, 3614-3617.

11. Svare A, Nilsen, TIL, Bjøro, T, Åsvold BO, Langhammer, A. Serum TSH related to measures of body mass: longitudinal data from the HUNT Study, Norway. Clin. Endocrinol. 2011, 74, 769-775.

12. Alexander EK, Pearce, EN, Brent, GA, Brown RS, Chen H, Dosiou, C, et al. 2017 Guidelines of the American Thyroid Association for the Diagnosis and Management of Thyroid Disease During Pregnancy and the Postpartum. Thyroid. 2017, 27, 315-389.

13. Pop VJ, Biondi B, Wijnen HA, KuppensSM, LVader H. Maternal thyroid parameters, body mass index and subsequent weight gain during pregnancy in healthy euthyroid women. Clin. Endocrinol. 2013, 79, 577-583.

14. Kahr MK., Antony KM, DelBeccaro M, Hu M, Aagaard KM, Suter M.A. Increasing maternal obesity is associated with alterations in both maternal and neonatal thyroid hormone levels. Clin. Endocrinol. 2016, 84, 551-557.

15. Zhou B, ChenY, Cai.WQ, Liu L, and Hu XJ. Effect of Gestational Weight Gain on Associations Between Maternal Thyroid Hormones and Birth outcomes. Frontiers in Endocrinology, 2020, 11, 610-618.

16. Thangaratinam S, Tan A, Knox E, Kilby MD, Franklyn J, Coomarasamy A. Association between thyroid autoantibodies and miscarriage and preterm birth: meta-analysis of evidence. BMJ. 2011, 342, d2616.

17. Wang ZY, Jin W, Zhu ZN, Cui XY, Song Q, Shi ZH, et al. Relationship of household cooking salt and eating out on iodine status of pregnant women in environmental iodine deficient coastal areas of China. Br J Nutr 2020, 124(9), 1-21.

18. Holmes H, Palacios C, Wu Y, Banna J. Effect of a Short Message Service Intervention on Excessive Gestational Weight Gain in a Low-Income Population: A Randomized Controlled Trial. Nutrients. 2020, 12, 1428.

19. Norris T, Seaton SE, Manktelow BN, Baker PN, Kurinczuk JJ, Field D, et al. Updated birth weight centiles for England and Wales. Archives of Disease in Childhood - Fetal and Neonatal Edition. 2018, 103, F577-F582. 
20. Collares FM, Korevaar TIM, Hofman A, Steegers EAP, Peeters RP, Jaddoe VWV, et al. Maternal thyroid function, prepregnancy obesity and gestational weight gain-The Generation R Study: A prospective cohort study. Clin. Endocrinol. 2017, 87, 799-806.

21. Haddow JE, Craig WY, Neveux LM, Haddow HRM, Palomaki GE, Lambert-Messerlian G, et al. Implications of High Free Thyroxine (FT4) Concentrations in Euthyroid Pregnancies: The FaSTER Trial. The Journal of Clinical Endocrinology \& Metabolism. 2014, 99, 2038-2044.

22. Derakhshan A, Peeters RP, Taylor PN, Bliddal S, Carty DM, Meems M, et al. Association of maternal thyroid function with birthweight: a systematic review and individual-participant data meta-analysis. Lancet Diabetes Endocrinol 2020, 8, 501-510

23. Medici M, Timmermans S, Visser W, de Muinck Keizer-Schrama SMPF, Jaddoe VWW, Hofman A, et al. Maternal Thyroid Hormone Parameters during Early Pregnancy and Birth Weight: The Generation R Study. The Journal of Clinical Endocrinology \& Metabolism. 2013, 98, 59-66.

24. Zhang C, Yang X, Zhang Y, Guo F, Yang S, Peeters RP, et al. Association Between Maternal Thyroid Hormones and Birth Weight at Early and Late Pregnancy. The Journal of Clinical Endocrinology \& Metabolism. 2019, 104, 5853-5863.

25. Vrijkotte TGM, Hrudey EJ, Twickler MB. Early Maternal Thyroid Function During Gestation Is Associated With Fetal Growth, Particularly in Male Newborns. The Journal of Clinical Endocrinology \& Metabolism. 2017, 102, 1059-1066.

26. Kiserud, Benachi A, Hecher K, Perez RG, Carvalho J, Piaggio G, et al. The World Health Organization fetal growth charts: concept, findings, interpretation, and application. Am. J. Obstet. Gynecol. 2018, 218, S619-S629.

27. Forhead AJ, Fowden AL. Thyroid hormones in fetal growth and prepartum maturation. J. Endocrinol. 2014, 221, R87-R103.

28. Männistö T, Vääräsmäki M, Pouta A, Hartikainen A, Ruokonen A, Surcel H, et al. Perinatal Outcome of Children Born to Mothers with Thyroid Dysfunction or Antibodies: A Prospective Population-Based Cohort Study. The Journal of Clinical Endocrinology \& Metabolism. 2009, 94, 772-779.

29. Duntas LH, Orgiazzi J, Brabant G. The interface between thyroid and diabetes mellitus. Clin. Endocrinol. 2011, 75, 1-9.

30. Belkacemi L, Nelson DM, Desai M, Ross MG. Maternal Undernutrition Influences Placental-Fetal Development1. Biol. Reprod. $2010,83,325-331$.

31. Ritter MJ, Amano I, Hollenberg AN. Thyroid Hormone Signaling and the Liver [published online ahead of print, 2020 Apr 28]. Hepatology. 2020.

32. GLINOER D, RIAHI M, GRON J, KINTHAERT AJ. Risk of Subclinical Hypothyroidism in Pregnant Women with Asymptomatic Autoimmune Thyroid Disorders. Journal of Clinical Endocrinology and Metabolism. 1994, 1, 197-204.

33. Negro R, Formos G, Mangieri T, Pezzarossa A, Dazzi D, Hassan H. Levothyroxine Treatment in Euthyroid Pregnant Women with Autoimmune Thyroid Disease: Effects on Obstetrical Complications. The Journal of Clinical Endocrinology \& Metabolism. 2006, 91, 2587-2591.

34. Aune D, Saugstad OD, Henriksen T, Tonstad S. Maternal Body Mass Index and the Risk of Fetal Death, Stillbirth, and Infant Death. JAMA. 2014, $311,1536$.

35. Yu Z, Han S, Zhu J, Sun X, Ji C, Guo X. Pre-pregnancy body mass index in relation to infant birth weight and offspring overweight/obesity: a systematic review and meta-analysis. PLoS One. 2013, 8, e61627. 